\title{
Contribuição da Apnéia Obstrutiva do Sono para o Estresse Oxidativo da Obesidade
}

\section{artigo original}

\author{
AnNa Myrna Jaguaribe de Lima \\ Cléla M. Ribeiro Franco \\ Céla M. M. Barbosa de Castro \\ Alice de ANdRAde BezerRa \\ LUIz AtaÍde JR. \\ Alfredo Halpern
}

Universidade de São Paulo (USP),

São Paulo, Brasil

\section{RESUMO}

Objetivo: O objetivo do trabalho foi verificar a influência da apnéia obstrutiva do sono (AOS) sobre o estresse oxidativo da obesidade e o efeito do pressão positiva de vias aéreas (CPAP) nestes pacientes. Métodos: Os pacientes com IMC $\geq 30 \mathrm{~kg} / \mathrm{m}^{2}$ foram divididos em: a) grupo 1: dez indivíduos sem AOS; b) grupo 2: dez portadores de AOS que não fizeram o uso do CPAP; e c) grupo 3: nove portadores de AOS que fizeram uso do CPAP durante dois meses. Resultados: $O$ grupo 3 apresentou, após o uso do CPAP, redução na produção de superóxido (SO) $\left[13,2(10,3-19,6)\right.$ versus $10,5(5,8-11,8)$ nmoles $O_{2}-12 \times 10^{6}$ PMN] e aumento na síntese de nitritos e nitratos séricos $[24,5(16,7-33,5)$ versus $49,5(39,3-58,1) \mu \mathrm{M}]$. Também foi verificada correlação positiva entre o índice apnéia-hipopnéia (IAH) e a produção de $S O(r=0,726)$ e negativa entre o IAH e os níveis de nitritos e nitratos séricos $(r=-0,867)$. Conclusões: $O$ estresse oxidativo presente na obesidade é exacerbado pela AOS e o uso do CPAP promove aumento nos níveis de nitritos e nitratos séricos, bem como reduções na produção de SO neste pacientes. (Arq Bras Endocrinol Metab 2008; 52/4:668-676)

Descritores: Apnéia obstrutiva do sono; Obesidade; Estresse oxidativo.

\section{ABSTRACT}

Obstructive Sleep Apnea Contribution to Oxidative Stress in Obesity.

Objective: The aim of this paper was to check the influence of obstructive sleep apnea (OSA) on obesity oxidative stress and CPAP (Continuous Positive Airway Pressure) effect on oxidative stress and in these patients. Methods: Twenty nine male patients considered obese $\left(\mathrm{BMI} \geq 30 \mathrm{~kg} / \mathrm{m}^{2}\right)$ were divided into 3 groups: a) Group I: 10 OSA free patients (apnea-hipopnea index (AHI) $\leq$ 5); b) Group 2: 10 with moderate to serious OSA (AHI $\geq 20$ ); c) Group 3: 9 with OSA from moderate to serious $\left(A H I \geq 20\right.$ ) using $C P A P_{,}$, minimum 4 hours/ night for 2 months. Results: Significant differences before and after CPAP usage were observed in group 3 in the following variables: reduction of superoxide (SO) production $\left[13,2(10,3-19,6)\right.$ vs. $10,5(5,8-11,8)$ nmoles $\mathrm{O}_{2}^{-} / 2 \times 10^{6}$ $\mathrm{PMN}]$ and increase in serum nitrite/nitrates levels $[24,5(16,7-33,5)$ vs. 49,5 $(39,3-58,1) \mu \mathrm{M}]$. Positive correlation between Apnea-Hypopnea Index (AHI) and $\mathrm{SO}(r=0,726)$ and negative correlation was observed between $\mathrm{AHI}$ and serum nitrite/nitrates levels $(r=-0,867)$. Conclusions: In conclusion, oxidative stress present in obesity is elevated by OSA and CPAP treatment can rise the levels of $\mathrm{SO}$ and can decrease serum nitrite/nitrates present in obese patients with OSA. (Arq Bras Endocrinol Metab 2008; 52/4:668-676)

Recebido em 11/12/2007 Aceito em 03/04/2008
Keywords: Obstructive sleep apnea; Obesity; Oxidative stress. 


\section{INTRODUÇÃO}

A APNÉIA OBSTRUTIVA DO SONO (AOS) é uma síndrome caracterizada por colapsos repetitivos das vias aéreas superiores (VAS) durante o sono, e a sua classificação é fundamentada no índice apnéia/hipopnéia (IAH) que quantifica o número total de apnéias e/ou hipopnéias por hora de sono $(1,2)$. A apnéia é definida como a interrupção total do fluxo aéreo por intervalo $\geq 10 \mathrm{~s}$ e a hipopnéia é a interrupção parcial $(\geq 50 \%)$ do fluxo aéreo. Ambas devem estar associadas à queda da saturação da hemoglobina de, no mínimo, $4 \%$ e/ou ao despertar do paciente $(3,4)$. De acordo com a classificação, considerase: a) AOS leve: $5>$ IAH < 15 eventos por hora; b) AOS moderada: $15>$ IAH $<30$ eventos por hora: c) AOS grave: $\mathrm{IAH}>30$ eventos por hora $(5,6)$.

A AOS é uma doença de alta prevalência, acometendo, segundo Young, Peppard e Gottlieb (6), 5\% da população ocidental. É mais freqüente em homens (4\%) do que em mulheres (2\%), até a menopausa, quando esses números se igualam (7). Tanto os indivíduos idosos quanto os obesos têm maior propensão a apresentar a $\operatorname{AOS}(8,9)$.

A terapia com pressão positiva de vias aéreas (CPAP) no tratamento da AOS é largamente utilizada e sua eficácia já foi comprovada por vários estudos, sendo considerada a principal escolha no tratamento da AOS (10-12). Muitas investigações têm sido realizadas a respeito dos efeitos do CPAP em várias situações patológicas associadas com a AOS, como alteração das funções neuroendócrinas (13), disfunção endotelial $(14,15)$, níveis de resistina, grelina e leptina $(9,16,17)$ e hiperatividade simpática (18).

Existe forte relação entre a AOS e o surgimento de doenças cardiovasculares e um dos fatores é o aumento do estresse oxidativo presente em ambas. As alterações repetidas na saturação sangüínea, presentes nos episódios de apnéia/hipopnéia e hipóxia-reoxigenação da AOS são similares ao quadro da injúria isquemia-reperfusão tecidual, promovendo danos aos vasos sangüíneos quando o fluxo é restaurado, em razão do aumento da produção de radicais livres $(19,20)$. Estas espécies reativas de oxigênio reagem com ácidos nucléicos, lipídeos e proteínas e ainda reduzem a produção de nitritos e nitratos séricos, causando lesão endotelial, fator de risco fortemente associado às doenças cardiovasculares $(21,22)$.

Dessa forma, o objetivo do presente trabalho foi verificar o efeito do CPAP sobre o estresse oxidativo em pacientes obesos portadores de AOS.

\section{MATERIAL E MÉTODOS}

\section{Amostra e critérios de inclusão}

Foram inclusos na pesquisa 29 pacientes, classificados como obesos graus II e III (IMC $\left.\geq 30 \mathrm{~kg} / \mathrm{m}^{2}\right)$, do sexo masculino, divididos em três grupos - grupo 1: 10 indivíduos sem AOS ( $\mathrm{IAH} \leq 5)$, que não tiveram $\mathrm{AOS}$ diagnosticada após a realização da polissonografia; grupo 2: 10 portadores de AOS de moderada a grave (IAH $\geq 20$ ), que não fizeram uso do CPAP, por não se adaptarem ao aparelho ou por terem optado pelo tratamento cirúrgico, após o prazo de 2 meses; grupo 3: 9 portadores de AOS de moderada a grave (IAH $\geq 20$ ), que fizeram o uso do CPAP. Foram pacientes com indicação, acesso e adaptação (no mínimo 4 horas por noite, durante 2 meses) ao CPAP.

Foram excluídos da pesquisa os pacientes portadores de diabetes tipo 1 ou 2 , em processo inflamatório agudo ou crônico, portadores de apnéia do sono central ou respiração de Cheyne-Stokes, doença pulmonar obstrutiva crônica, asma, disfunções da tireóide e aqueles que estavam utilizando outra forma de tratamento para AOS (por exemplo: perda de peso, tonsilectomia) ou fazendo uso de corticoesteróides e $\beta$-agonistas ou bloqueadores.

Os indivíduos que concordaram em participar da pesquisa assinaram o termo de consentimento livre e esclarecido, de acordo com a Resolução CNS $\mathrm{n}^{\circ}$ 196/1996 do Ministério da Saúde, e aprovado pelo comitê de ética em pesquisa do Hospital das Clínicas da Faculdade de Medicina da Universidade de São Paulo (HC-FMUSP).

\section{Medidas antropométricas}

Foram mensurados no início e após dois meses, nos pacientes dos três grupos experimentais, peso $(\mathrm{kg})$ e altura $(\mathrm{m})$, utilizando a balança antropométrica (Filizola ${ }^{\circledR}$ ) e o IMC (peso/altura $\left.{ }^{2}\right)\left(\mathrm{kg} / \mathrm{m}^{2}\right)$.

\section{Polissonografia}

Os três grupos experimentais foram submetidos à polissonografia de noite inteira. Para realização deste exame, foi utilizado um sistema de polissonografia computadorizada (Healthdyne Technologies, Respironics, Alice $3^{\circledR}$ ) do Laboratório do Sono da Clínica Neurológica Luís Ataíde. Os parâmetros mensurados foram: eletrocardiograma (ECG), pressão arterial (PA), movimentos torácicos e abdominais (pletismografia), saturação peri- 
férica de oxigênio $\left(\mathrm{SpO}_{2}\right)$ (oximetria de pulso), fluxo aéreo nasal/bucal (termistores), posição corporal e ronco (microfone). Foram calculados, ainda, o número de apnéias, hipopnéias, o IAH e as quedas na $\mathrm{SpO}_{2}$, conforme descrito anteriormente.

\section{Uso do CPAP}

Após a polissonografia diagnóstica, foi realizada outra polissonografia com os pacientes portadores de AOS, a fim de titular (quantificar) a pressão do CPAP (Somnotron $41^{\circledR}$, Weinmann). Para cada paciente, foi administrada a pressão mínima suficiente para eliminar as apnéias, hipopnéias e roncos em todas as posições corporais e em todas as fases de sono. Inicialmente, foi imposta uma pressão de $0,4 \mathrm{kPa}$ (4 mbars) e, após cada 5 minutos (no mínimo) da ocorrência dos eventos restritivos, esta pressão foi aumentada em $0,1 \mathrm{kPa}$ ( 1 mbar). Se estes eventos cessassem nos próximos 30 minutos, a pressão era reduzida em $0,1 \mathrm{kPa}$ ( 1 mbar), a cada 10 minutos, até que os eventos obstrutivos se manifestassem novamente e todo o procedimento fosse repetido.

\section{Obtenção de monócitos a partir do sangue periférico}

Os monócitos foram obtidos a partir da coleta de sangue venoso, com EDTA, entre 7 e 8 horas. O sangue obtido foi diluído na proporção de 1:2 em meio de cultura RPMI 1640, estéril, à temperatura ambiente de $22{ }^{\circ} \mathrm{C}$ a $25{ }^{\circ} \mathrm{C}$ (15 ml de sangue $+15 \mathrm{ml}$ de RPMI 1640). Aos $30 \mathrm{ml}$ da suspensão foram adicionados $15 \mathrm{ml}$ de histopaque (1077 - SIGMA) e todo o conteúdo foi centrifugado por 30 minutos a $1.400 \mathrm{rpm}\left(20^{\circ} \mathrm{C}\right.$ a $\left.80{ }^{\circ} \mathrm{C}\right)$.

Logo após, o plasma foi aspirado e, então, coletouse a camada formada pelas células (PBMC) que foi transferida para outro tubo de ensaio. Foram adicionados 15 $\mathrm{ml}$ de RPMI 1640 e centrifugados por 10 minutos nas mesmas condições anteriores. O sobrenadante foi aspirado e desprezado. Posteriormente, foram realizadas duas novas lavagens com RPMI 1640, centrifugando 5 minutos a cada lavagem. Mais uma vez o sobrenadante foi desprezado e o sedimento ressuspendido em $2 \mathrm{ml} \mathrm{do}$ meio de cultura RPMI 1640 completo, contendo 3\% de soro fetal bovino e antibióticos (penicilina $100 \mathrm{U} / \mathrm{ml}$ e estreptomicina $100 \mu \mathrm{g} / \mathrm{ml}$ ). Dessa suspensão, foi feita a contagem em câmara de Neubauer acrescendo-se a alíquota da suspensão de células e o corante azul tripan na diluição 1:10. Esse corante é usado para fazer a contagem das células e avaliar a sua viabilidade. A concentra- ção celular foi ajustada para $2 \times 106$ células em $2 \mathrm{ml}$ do meio. As células foram colocadas em placas do tipo Falcon com 6 poços de 35 milímetros de diâmetro cada, nos quais foram dispensados os $2 \mathrm{ml}$ da suspensão $(2 \times$ 106 células por $2 \mathrm{ml}$ de meio de cultura RPMI 1640 completo) em cada poço. Após esse tempo, os poços foram lavados com meio de cultura RPMI 1640 para retirada das células não aderentes. $\mathrm{O}$ material permaneceu por mais 1 hora na incubadora em atmosfera úmida, temperatura de $37{ }^{\circ} \mathrm{C}$ e $5 \%$ de $\mathrm{CO}_{2}$.

\section{Produção do ânion superóxido}

Esse ânion pode ser detectado a partir da sua capacidade em reduzir quimicamente um composto aceptor de elétrons. Utilizou-se, para esse fim, o ferricitocromo C (30 $\mathrm{mg} / \mathrm{ml} \mathrm{em} \mathrm{HBSS}, 2,4 \times 10^{-3} \mathrm{M}$, ferricitocromo C de mitocôndria de eqüino, tipo III, da Sigma Chemical Company, de St. Louis, MO, Estados Unidos). Foi necessária, ainda, a utilização da enzima, superóxido dismutase (enzima superóxido dismutase de eritrócitos bovinos - SOD, Sigma Chemical Company ${ }^{\circledR}$ ) contendo $3.000 \mathrm{u} / \mathrm{mg}$ de proteína em solução final de $3 \mathrm{mg} / \mathrm{ml}$ em água destilada.

Para a avaliação da produção de superóxido formado, foram preparados dois sistemas de análise descontínua com uma primeira avaliação logo após o início da estimulação das células com acetato miristato de forbol (PMA, da Sigma Chemical Company) e uma segunda avaliação após 2 horas.

Para o preparo desses sistemas, foram usados monócitos em cultura, adicionando-se SOD ao primeiro sistema (controle negativo), e, ao segundo, água destilada (controle positivo). Estes foram mantidos em incubadora com atmosfera úmida a $5 \%$ de $\mathrm{CO}_{2}$ a $37^{\circ} \mathrm{C}$ por 10 minutos para ativação da SOD. Ao meio foi acrescido citocromo C e PMA (o PMA foi preparado em solução concentrada de $3.000 \mu \mathrm{g} / \mathrm{ml}$ em dimetilsulfóxido de sódio (DMSO, SIGMA), diluído para uma concentração de $2 \mu \mathrm{g} / \mathrm{ml}$ em $2.145 \mathrm{ml}$ de solução salina balanceada de Hanks (HBSS GIBCO) e colocado nos poços da placa de cultura.

No segundo sistema, contendo água destilada, também foram adicionados citocromo C e PMA. Em seguida, amostras de $600 \mu \mathrm{l}$ foram retiradas concomitantemente de cada sistema e postas em tubos Eppendorf. A primeira alíquota recolhida relativa ao tempo zero de cada sistema correspondeu ao branco. A amostra subseqüente foi coletada em intervalo de tempo de 2 horas nas mesmas condições. 


\section{Determinação espectrofotométrica}

Ao término da última coleta, as amostras foram centrifugadas a $10.000 \mathrm{rpm}$ por 5 minutos à temperatura ambiente, em microcentrífuga (25.000 g - rotor Ra-1M Kubota).

O sobrenadante foi levado para determinação do grau de redução de ferrocitocromo $\mathrm{C}$ (proporcional à produção do radical superóxido) usando-se cubeta de 1 $\mathrm{ml}$ de quartzo e espectofotômetro com comprimento de onda ajustado para a faixa do vermelho $(550 \mathrm{~nm})$.

Os resultados finais foram expressos após o agrupamento dos respectivos pontos para cada tempo de coleta de cada paciente. O cálculo final foi dado pela fórmula: Cálculo $[\mathrm{O} 2-]=\mathrm{K} \times \mathrm{DO} \times \mathrm{V}$. Em que: $\mathrm{K}$ : constante calculada para o volume final em cada poço; DO: densidade ótica; e V: volume da amostra.

No presente estudo a constante $\mathrm{K}$, calculada para o volume final em cada poço foi de 205,49.

\section{Produção de óxido nítrico (NO) em monócitos cultivados e tratados com lipopolissacarídeo de E. coli (LPS)}

Em cada grupo a concentração foi ajustada para $1 \times 10^{6}$ células em $1 \mathrm{ml}$ de meio de cultura em cada poço da placa. Em seguida, as células foram tratadas na dose de $10 \mu \mathrm{g} / \mathrm{mL}$ de LPS por 24 horas. A avaliação da liberação de NO foi realizada utilizando-se o método de GRIESS. Este método quantifica indiretamente a produção de NO pela determinação de nitritos e nitratos acumulados no sobrenadante das células, após o tratamento. As amostras e o padrão (NaNO2 $1 \mathrm{mM}$ - 251-4 Sigma Chemical, St. Louis, MO; EUA) foram colocados em placa de vinil nas concentrações determinadas $(1,56 \mu \mathrm{M}$ a 50 $\mu \mathrm{M})$, diluídas em RPMI 1640 (50 $\mu$ l por poço). Em se- guida, foram adicionados $50 \mu \mathrm{l}$ do reagente de GRIESS (1 g de sulfanilamide, Sigma 9251; 0,1 g de N-(Naphthyl) Ethylenediamine Dihydrochloride - Sigma 5889;2,5 ml de ácido fosfórico PA e água destilada qsp $100 \mathrm{ml}$ ). A placa foi incubada por 10 minutos ao abrigo da luz. A leitura foi realizada a $540 \mathrm{~nm}$ em leitor de Elisa (Dynatech MR 5000). O limiar de sensibilidade do teste foi de $1,56 \mu \mathrm{M}$.

\section{Análise estatística}

$\mathrm{Na}$ verificação das diferenças inter e intragrupos, foram realizados os testes de Wilcoxon e Mann-Whitney. Para o estabelecimento das correlações foi utilizado o coeficiente de Spearman. Os resultados foram apresentados como média e erro-padrão. $\mathrm{O}$ valor de $\mathrm{p}<0,05$ foi considerado como nível de significância estatística.

\section{RESULTADOS}

Na Tabela 1, pode-se observar as características dos três grupos experimentais. Houve diferença significativa na saturação mínima de oxigênio e no IAH ao se comparar os grupos de obesos com AOS com o grupo de obesos sem AOS.

Ao se analisar a Figura 1, verifica-se que a produção de superóxido foi superior nos grupos 2 e 3 quando comparada ao do grupo 1 , enquanto a produção de nitritos e nitratos séricos foi inferior nos grupos 2 e 3 quando comparada ao grupo 1.

Também foram identificadas diferenças significativas no grupo 3 , antes e após 2 meses do uso do CPAP, na produção de superóxido e na produção de nitritos $\mathrm{e}$ nitratos séricos. Verificou-se, ainda, que no grupo 3, após o uso do CPAP, a produção de nitritos e nitratos

Tabela 1. Características da amostra.

\begin{tabular}{cccc}
\hline & $\begin{array}{c}\text { Obesos sem AOS } \\
(\text { Grupo 1) } \\
\mathbf{n}=10\end{array}$ & $\begin{array}{c}\text { Obesos com AOS sem CPAP } \\
\text { (Grupo 2) } \\
\mathbf{n}=10\end{array}$ & $\begin{array}{c}\text { Obesos com AOS com CPAP } \\
\text { (Grupo 3) } \\
\mathbf{n}=\mathbf{9}\end{array}$ \\
\hline Idade (anos) & $56,8 \pm 4,7$ & $57 \pm 3,3$ & $55,8 \pm 3,5$ \\
\hline $\mathrm{IMC}\left(\mathrm{kg} / \mathrm{m}^{2}\right)$ & $33,1 \pm 2,5$ & $34,1 \pm 1,3$ & $37,3 \pm 4,9$ \\
\hline $\mathrm{AH}($ eventos/h) & $3,6 \pm 0,1$ & $29,5 \pm 3,7^{*}$ & $29,9 \pm 2,9^{*}$ \\
\hline $\mathrm{SaO}_{2}(\%)$ & $84,5 \pm 3,2$ & $65,3 \pm 4,7^{\star}$ & $71,3 \pm 2,5^{*}$ \\
\hline $\mathrm{FC}(\mathrm{bpm})$ & $92,0 \pm 1,9$ & $99,6 \pm 2,2^{*}$ & $100,8 \pm 2,5^{*}$ \\
\hline
\end{tabular}

Os resultados estão mostrados como média \pm erro-padrão. IMC = índice de massa corpórea; IAH = índice apnéia-hipopnéia; $\mathrm{SaO}_{2}-=$ saturação mínima de oxigênio: $\mathrm{FC}=$ freqüência cardíaca. ${ }^{*} \mathrm{p}<0,05=$ diferença significativa em relação ao grupo 1. 
séricos e de superóxido foram similares à do grupo 1 (Figura 2).

Ao se avaliar a relação entre o IAH e a produção de superóxido dos pacientes obesos com AOS (Figura
3), verificou-se a existência de correlação positiva entre as variáveis, mostrando que quanto maiores os valores do IAH mais elevada era a produção de superóxido.

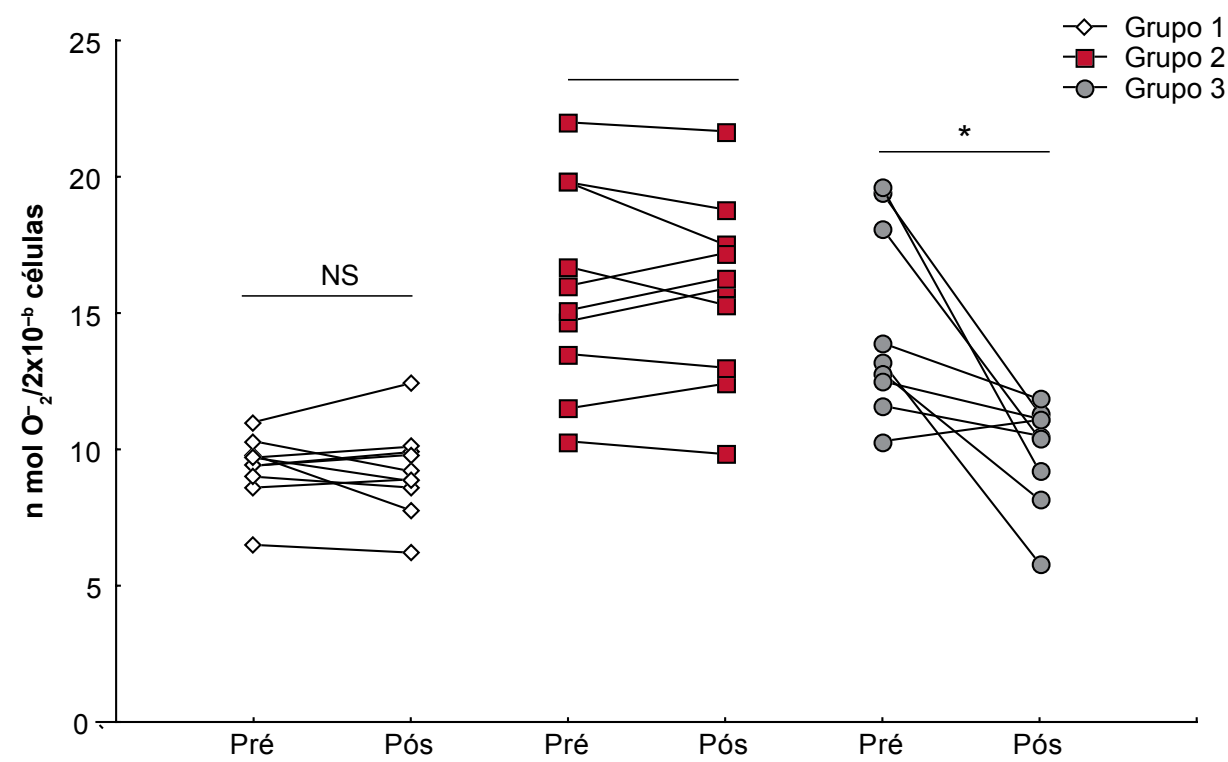

Figura 1. Produção de superóxido em indivíduos obesos sem AOS (grupo 1), obesos com AOS sem CPAP (grupo 2) e obesos com AOS com CPAP (grupo 3), antes (pré) e após 2 meses.

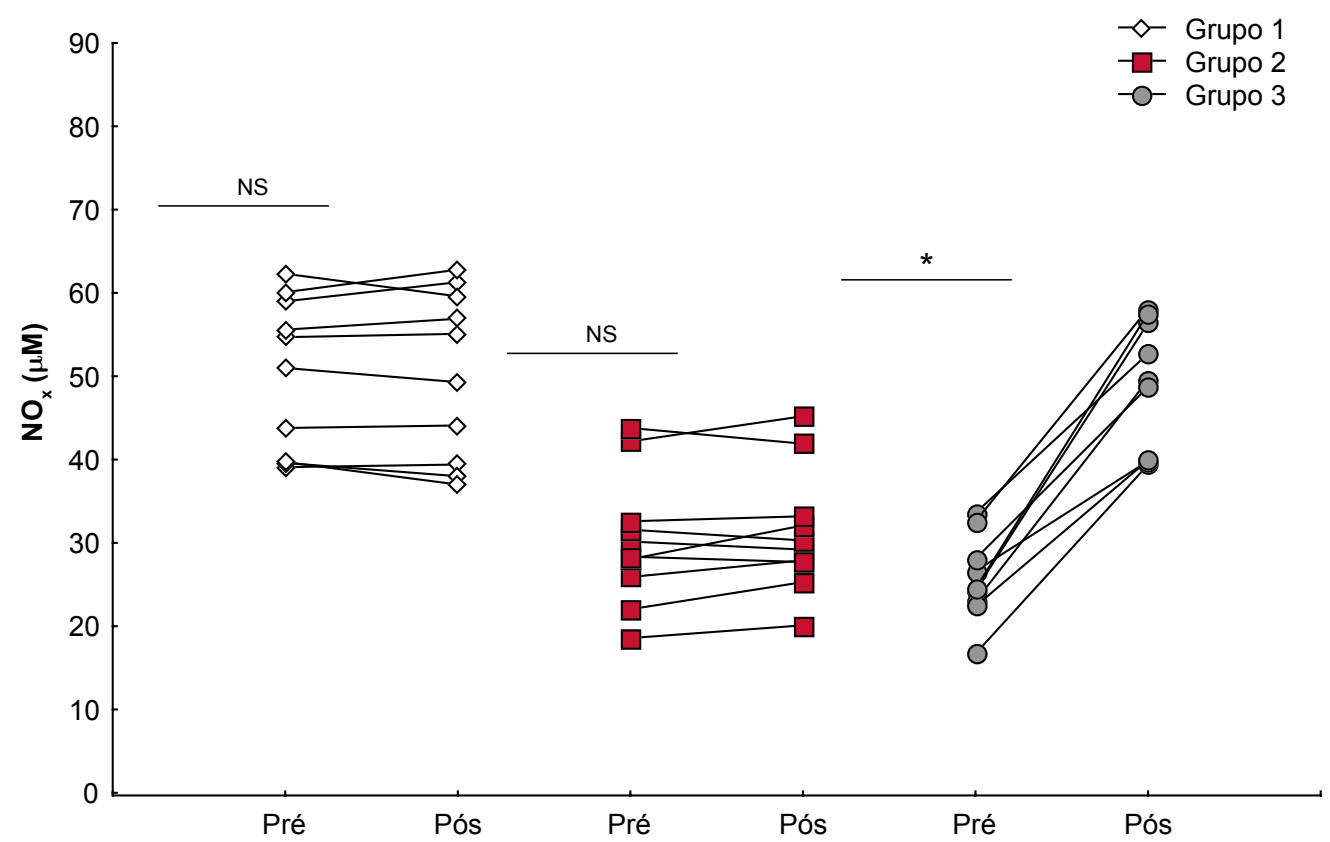

Figura 2. Níveis de nitritos e nitratos séricos em indivíduos obesos sem AOS (grupo 1), obesos com AOS sem CPAP (grupo 2) e obesos com AOS com CPAP (grupo 3), antes (pré) e após 2 meses (pós). 
Na Figura 4, está demonstrada a relação entre o IAH e a produção de nitritos e nitratos séricos dos pacientes obesos com AOS, na qual há uma correlação significativa entre as variáveis analisadas, mostrando que quanto maiores os valores de IAH menor era a produção de nitritos e nitratos séricos.

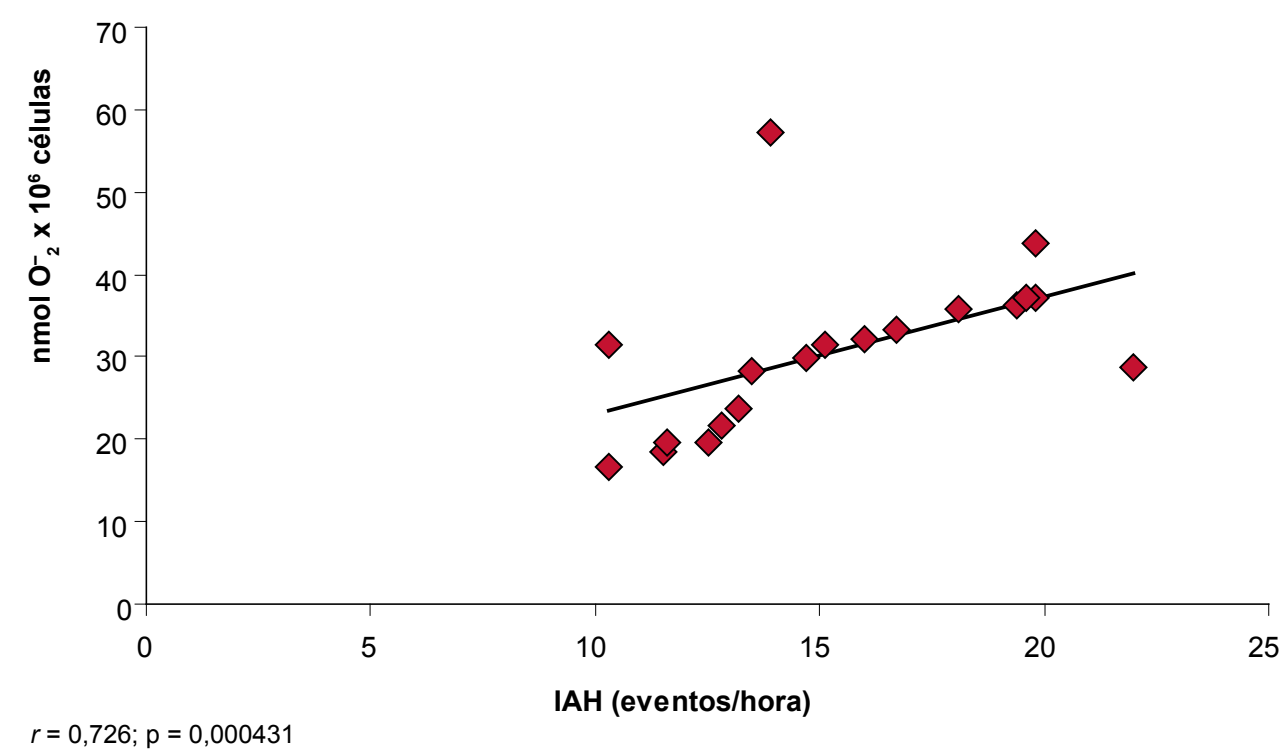

Figura 3. Correlação entre o IAH e a produção de superóxido em indivíduos obesos portadores de AOS (grupos 2 e 3, em níveis basais).

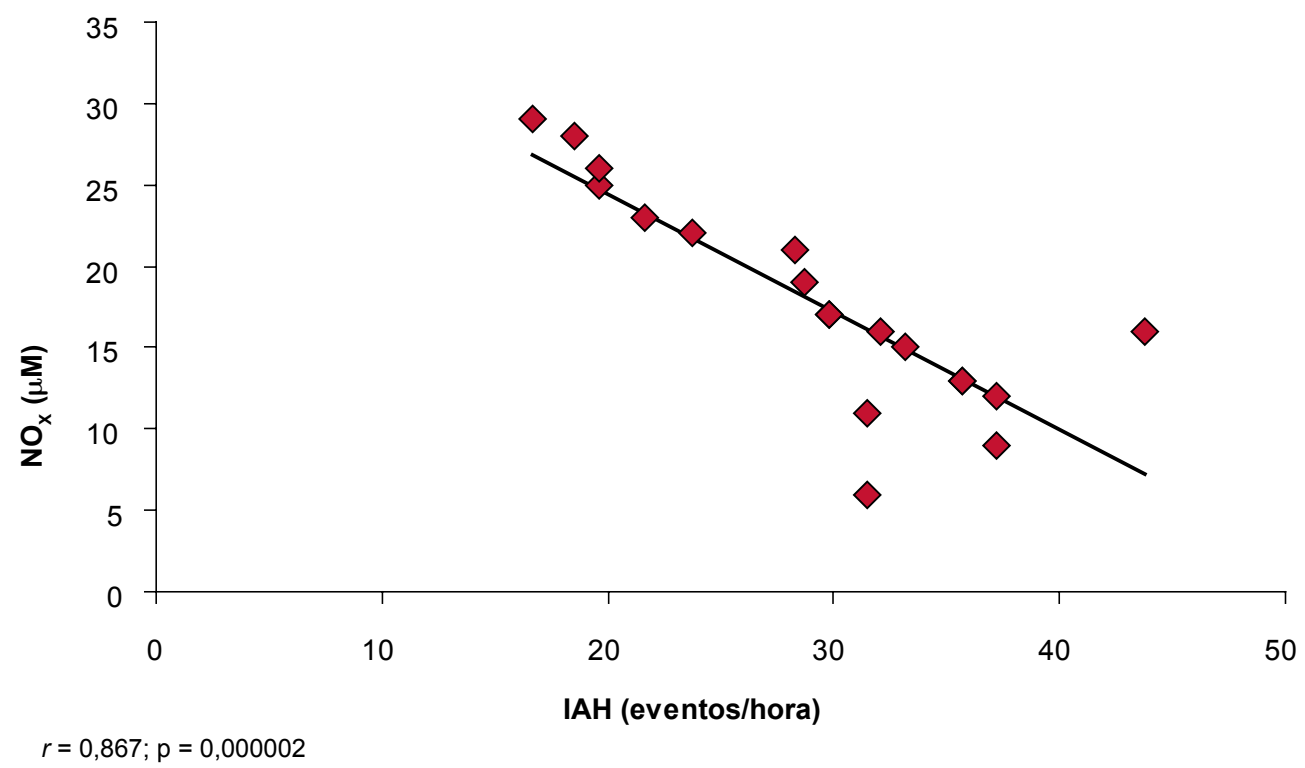

Figura 4. Correlação entre o IAH e os níveis de nitritos e nitratos séricos (NOx) em indivíduos obesos portadores de AOS (grupos 2 e 3, em níveis basais). 


\section{DISCUSSÃo}

A AOS está relacionada à ocorrência de doenças cardiovasculares e patogênese das condições de morbidade, como hipertensão, resistência à insulina e dislipidemia. Acredita-se que os principais responsáveis pelas disfunções metabólicas e cardiovasculares induzidas pela AOS sejam a hipoxemia intermitente e o aumento do tônus simpático. A obesidade também está associada com o aumento da prevalência de doenças cardiovasculares, além do que indivíduos obesos apresentam maior predisposição para o desenvolvimento de AOS.

A função endotelial é de extrema importância para o equilíbrio do tônus vasomotor e para a manutenção da fluidez do sangue no vaso. Para exercer essas funções, é necessário que o endotélio promova a liberação de substâncias vasoativas, como os nitritos e os nitratos séricos (NO). Efeitos sobre a síntese e a biodisponibilidade do NO, principal substância vasodilatadora derivada do endotélio, alterarão a vasomotricidade e a função endotelial (23). Situações como a obesidade e a AOS promovem alterações na funcionalidade do endotélio, perturbando o equilíbrio na liberação de substâncias vasocontritoras e vasodilatadoras e causando disfunção endotelial (24).

No presente estudo, os indivíduos obesos com AOS e sem AOS apresentaram redução nos níveis de $\mathrm{NO}$ e aumento na síntese de superóxido ( $\mathrm{SO}$ ), alterações revertidas após o uso de CPAP durante 2 meses (Figuras 1 e 2). A produção de NO reproduz o relaxamento vascular dependente do endotélio. Como o tempo de meiavida dos nitritos e nitratos (derivados do NO) é de 4 a 7 horas, a mensuração desses elementos, logo após o despertar, reflete a produção de NO durante a noite, período no qual os pacientes apnéicos apresentam os episódios recorrentes de hipóxia/reoxigenação (25).

Schulz e cols. (26) estudaram a produção de NO em três grupos: indivíduos saudáveis, indivíduos com AOS e indivíduos com outras doenças. Os autores verificaram que os pacientes com AOS apresentavam níveis reduzidos de NO, quando comparados aos outros dois grupos. Também foi observado neste mesmo estudo, que o uso do CPAP, tanto a curto (2 dias) quanto a longo prazo ( 5,5 meses), promovia elevação nos níveis de NO. Esses dados mostram o efeito direto da redução e/ou eliminação dos episódios de hipóxia/reoxigenação pelo CPAP sobre a produção de NO.

Outro estudo, realizado por Ip e cols. (27) em indivíduos saudáveis e portadores de AOS, também verificou níveis inferiores de NO nos indivíduos com AOS, quando comparados ao grupo-controle. Após três meses do uso do CPAP, houve aumento significativo da síntese de NO nos pacientes portadores de AOS.

Não há relatos na literatura de estudos sobre a produção de NO e SO em indivíduos obesos com AOS, mas já é comprovado que a obesidade é um fator que aumenta o estresse oxidativo. No trabalho publicado por $\mathrm{Fu}^{-}$ rukawa e cols. (28), foi relatado que o estresse oxidativo em indivíduos obesos é responsável pelo estabelecimento da síndrome metabólica por intermédio dos seguintes mecanismos: a) o aumento na produção de radicais livres leva ao desequilíbrio na produção das adipocitocinas; e b) o aumento seletivo das espécies reativas de oxigênio nas regiões em que há acúmulo de gordura leva à elevação do estresse oxidativo sistêmico.

Um novo e importante achado deste estudo é que o equilíbrio antioxidante/oxidante na AOS é perturbado, independente da presença da obesidade, já que os pacientes obesos portadores de AOS apresentaram níveis de $\mathrm{NO}$ inferiores e de $\mathrm{SO}$ superiores àqueles encontrados nos indivíduos obesos sem AOS.

Em nosso estudo, a produção de $\mathrm{NO}$ em indivíduos obesos com AOS mostrou correlação com o IAH, reforçando o papel dos eventos de hipóxia/reoxigenação sobre o estresse oxidativo (Figura 4). Observou-se também que quanto maior a gravidade da AOS, caracterizada por valores elevados de IAH, menor era a produção de NO.

Ip e cols. (27) mostraram a correlação inversa entre o IAH e a produção de NO nos indivíduos com AOS, relação esta que permaneceu mesmo após terem sido feitos ajustes para o IMC, indicando que a AOS produz alterações na síntese de nitritos e nitratos séricos, independente da obesidade.

A redução na produção de NO observada na AOS também é acompanhada de prejuízo à função vascular, restabelecida após o uso do CPAP, como foi relatado por Imadojemu e cols. (29). Eles demonstraram que a hiperemia reativa decorrente de oclusão do fluxo arterial do antebraço durante 10 minutos estava atenuada e foi incrementada após o uso do CPAP, em períodos de duas semanas a 24 meses.

Quanto à produção das espécies reativas de oxigênio capazes de inativar o $\mathrm{NO}$, verificou-se que a síntese de SO se apresentava aumentada nos indivíduos obesos com AOS, quando comparados aos obesos sem AOS. Após a utilização do CPAP por 2 meses, por serem regularizados os eventos de hipóxia/reoxigenação, a produção de SO apresentou supressão (Figura 1). 
O aumento da síntese de SO na AOS apresenta correlação com a redução nos níveis de ON e com possíveis alterações na homeostase do tônus vasomotor com conseqüente lesão endotelial, e é influenciado pelos seguintes elementos: a) a hipoxemia intermitente funciona como ativadora dos neutrófilos polimorfonucleares, que aderem ao endotélio e liberam as espécies reativas de oxigênio, induzindo ao aumento na produção de radicais livres. Esta resposta depende do grau e da duração da hipóxia (30); b) a hiperatividade simpática presente na AOS, que promove elevação nos níveis das catecolaminas plasmáticas, levam ao processo de auto-oxidação, elevando a síntese das espécies reativas de oxigênio (31); c) fragmentação do sono, com privação do sono REM (32). No estudo realizado por Carpagnano e cols. (33) foi verificada a correlação negativa entre o tempo de sono REM na duração total do sono e a produção de 8 -isoprostano, um marcador de estresse oxidativo; d) processo inflamatório da VAS e congestão nasal, associados à elevação de fatores inflamatórios como a IL-6 e o TNF- $\alpha$, elementos associados com o aumento do estresse oxidativo.

A inativação do óxido nítrico pelos radicais livres de oxigênio e a ativação dos receptores de angiotensina II e tromboxane A2 $\left(\mathrm{TXA}_{2}\right)$ aumentam a geração de endotelina-1 (ET-1) e podem ocasionar vasoconstrição e disfunção endotelial (34). Além disso, a vasodilatação independente do endotélio também se mostrou comprometida nos pacientes com AOS, conforme foi demonstrado por Kato e cols. (35), em experimento realizado com indivíduos obesos portadores de AOS e saudáveis. Ao serem expostas à hipóxia, dependendo da duração do estímulo, as células endoteliais podem responder da seguinte maneira: a) a hipóxia transitória induz à modulação fisiológica e reversível do tônus vasomotor e do fluxo sangüíneo; b) a hipóxia crônica promove remodelação permanente dos vasos, com proliferação das células musculares lisas e fibrose.

De acordo com os resultados do presente trabalho, pode-se concluir que: a AOS aumenta o estresse oxidativo e, conseqüentemente, o processo inflamatório presente na obesidade; o uso do CPAP é capaz de melhorar as elevações na produção de superóxido e as reduções nos níveis de nitritos e nitratos séricos presentes nos pacientes obesos portadores de AOS.

\section{REFERÊNCIAS}

1. Olson EJ, Moore WR, Morgenthaler TI, Gay PC, Staats BA. Obstructive sleep apnea-hypopnea syndrome. Mayo Clin Proc. 2003;78:1545-52.

2. Hamilton GS, Solin P, Naughton MT. Obstructive sleep apnoea and cardiovascular disease. Intern Med J. 2004;34:420-26.

3. Redline S, Kapur VK, Sanders MH, Quan SF, Gottlieb DJ, Rapoport DM, et al. Effects of varying approaches for identifying respiratory disturbances on sleep apnea assessment. Am J Respir Crit Care Med. 2000;161:369-74.

4. Caples SM, Gami AS, Somers VK. Obstructive sleep apnea. Ann Intern Med. 2005;142:187-97.

5. American Academy of Sleep Medicine Task Force. Sleep-related breathing disorders in adults: recommendations for syndrome definition and measurement techniques in clinical research. Sleep. 1999;22:667-89.

6. Young T, Peppard P, Gottlieb D. Epidemiology of obstructive sleep apnea: a population health perspective. Am J Respir Crit Care Med. 2002;165:1217-39.

7. Bixler EO, Vgontzas AN, Lin H, Ten Have T, Rein J, Vela-Bueno $A$, et al. Prevalence of sleep-disordered breathing in women. Am J Respir Crit Care Med. 2002;163:608-13.

8. Bixler EO, Vgontzas AN, Ten Have T, Tyson K, Kales A. Effects of age on sleep apnea in men: I. Prevalence and severity. Am J Respir Crit Care Med. 1998;157:144-8.

9. Shimura R, Tatsumi K, Nakamura A, Kasahara Y, Tanabe N, Takiguchi $Y$, et al. Fat accumulation, leptin, and hypercapnia in obstructive sleep apnea-hypopnea syndrome. Chest. 2005; 127:543-9.

10. Wright J, Johns R, Watt I, Melville A, Sheldon T. Health effects of obstructive sleep apnoea and the effectiveness of continuous positive airways pressure: a systematic review of the research evidence. BMJ. 1997;314:851-60.

11. Jenkinson C, Davies RJ, Mullins R, Stradling JR. Comparison of therapeutic and subtherapeutic nasal continuous positive airway pressure for obstructive sleep CPAP in sleep apnoea: a randomised prospective parallel trial. Lancet. 1999;353:2100-5.

12. Hsu $A A L$, Lo C. Continuous positive airway pressure therapy in sleep apnoea. Respirology. 2003;8:447-54.

13. Yee B, Liu P, Phillips C, Grunstein R. Neuroendocrine changes in sleep apnea. Curr Opin Pulm Med. 2004;10:475-81.

14. Ip MS, Lam B, Chan LY, Zheng L, Tsang KW, Fung PC, et al. Circulating nitric oxide is suppressed in obstructive sleep apnea and is reversed by nasal continuous positive airway pressure. Am J Respir Crit Care Med. 2000:162:2166-71.

15. Schulz R, Mahmoudi S, Hattar K, Sibelius U, Olschewski H, Mayer K, et al. Enhanced release of superoxide from polymorphonuclear neutrophils in obstructive sleep apnea: impact of continuous positive airway pressure therapy. Am J Respir Crit Care Med. 2000;162:566-70.

16. Harsch IA, Koebnick C, Wallaschofski H, Ficker, JH, Lohmann T, Konturek PC. Resistin levels in patients with obstructive sleep apnoea syndrome - the link to subclinical inflammation? Med Sci Monit. 2004;10:CR510-15.

17. Harsch IA, Konturek PC, Koebnick C, Kuehnlein PP, Fuchs FS, Pour Schahin S, et al. Leptin and ghrelin levels in patients with obstructive sleep apnoea: effect of CPAP treatment. Eur Respir J. 2003;22:251-7.

18. Narkiewicz K, Somers SK. Sympathetic nerve activity in obstructive sleep apnoea. Acta Physiol Scand. 2003;177:385-90. 
19. McCord JM. Oxygen-derived free radicals in post-ischemic tissue injury. N Engl J Med. 1985;312:159-63.

20. McCord JM. The evolution of free radicals and oxidative Stress. Am J Med. 2000;108:652-9.

21. Yamauchi M, Nakano $H$, Maekawa J, Okamoto $Y$, Ohnishi $Y$, Suzuki T, et al. Oxidative stress in obstructive sleep apnea. Chest. 2005;127:1674-9.

22. Suzuki YJ, Jain V, Park AM, Day RM. Oxidative stress and oxidant signaling in obstructive sleep apnea and associated cardiovascular diseases. Free Radic Biol Med. 2006;40:1683-92.

23. Olusi SO. Obesity is an independent risk factor for plasma lipid peroxidation and depletion of erythrocyte cytoprotective enzymes in humans. Int $\mathrm{J}$ Obes Relat Metab Disord. 2002;26:1159-64.

24. Keaney JF Jr, Larson MG, Vasan RS, Wilson PW, Lipinska I, Corey D, et al. Framingham Study. Obesity and systemic oxidative stress: clinical correlates of oxidative stress in Framingham Study. Arterioscler Thromb Vasc Biol. 2003;23:434-9.

25. Viinikka L. Nitric oxide as a challenge for the clinical chemistry laboratory. Scand J Clin Lab Invest. 1996;56:577-81.

26. Schulz R, Mahmoudi S, Hattar K, Sibelius U, Olschewski $H_{\text {, }}$ Mayer K, et al. Enhanced release of superoxide from polymorphonuclear neutrophils in obstructive sleep apnea: impact of continuous positive airway pressure therapy. Am J Respir Crit Care Med. 2000;162:566-70.

27. Ip MS, Lam B, Chan LY, Zheng L, Tsang KW, Fung PC, et al. Circulating nitric oxide is suppressed in obstructive sleep apnea and is reversed by nasal continuous positive airway pressure. Am J Respir Crit Care Med. 2000;162:2166-71.

28. Furukawa S, Fujita T, Shimabukuro M, Iwaki M, Yamada Y, Nakajima $Y$, et al. Increased oxidative Stress in obesity and its impact on metabolic syndrome. J Clin Invest. 2004;114:1752-61.

29. Imadojemu VA, Gleeson K, Quraishi SA, Kunselman AR, Sinoway $\mathrm{LI}$, Leuenberger $\mathrm{U}$. Impaired vasodilator responses in obstructive sleep apnea are improved with continuous positive airway pressure therapy. Am J Respir Crit Care Med. 2002;165:950-3.

30. Khalid MA, Ashraf M. Direct detection of endogenous hydro$\mathrm{xyl}$ radical production in cultured adult cardiomyocyte during anoxia and reoxygenation: is the hydroxyl radical really the most damaging radical species? Circ Res. 1993;72:725-36.

31. Vargas F, Moreno JM, Wangensteen R, Rodriguez-Gomez I, Garcia-Estan J. The endocrine system in chronic nitric oxide deficiency. Eur J Endocrinol. 2007 Jan;156:1-12.

32. Glebocka A, Kossowska A, Bednarek M. Obstructive sleep apnea and the quality of life. J Physiol Pharmacol. 2006 Sep;57 Suppl 4:111-7.

33. Carpagnano GE, Kharitonov SA, Resta O, Foschino-Barbaro MP, Gramiccioni E, Barnes PJ. 8-Isoprostane, a marker of oxidative Stress, is increased in exhaled breath condensate of patients with obstructive sleep apnea after night and is a reduced by continuous positive airway pressure therapy. Chest. 2003; 124:1386-92.

34. Weiss JW, Liu MD, Huang J. Physiological basis for a causal relationship of obstructive sleep apnoea to hypertension. Exp Physiol. 2007;92:21-6.

35. Kato M, Roberts-Thomson P, Phillips BG, Haynes WG, Winnicki M, Accurso V, et al. Impairment of endothelium-dependent vasodilation of resistance vessels in patients with obstructive sleep apnea. Circulation. 2000; 21:2607-10.

\section{Endereço para correspondência:}

Anna Myrna Jaguaribe de Lima

Rua José Armando Machado, 91, apto. 304

51130-170 Boa Viagem, Recife, PE

E-mail: annamyrna@uol.com.br 